The University of San Francisco

USF Scholarship: a digital repository @ Gleeson Library | Geschke Center

“Systems Analysis and Design Toolkit Based on Work System Theory and Its Extensions

Steven Alter

Dominik Bork

Follow this and additional works at: https://repository.usfca.edu/at

Part of the Business Commons 


\title{
Systems Analysis and Design Toolkit Based on Work System Theory and Its Extensions
}

\author{
Steven Alter, University of San Francisco, USA \\ Dominik Bork, Faculty of Computer Science, University of Vienna, Vienna, Austria \\ iD https://orcid.org/0000-0001-8259-2297
}

\begin{abstract}
This article describes proposed content of an online toolkit for users of the work system modeling method (WSMM), an extension of the work system method (WSM), which was developed to help business professionals understand IT-reliant systems and collaborate with IT professionals. A summary of work system theory (WST) and WSM provides a background. A two-dimensional design space for modeling methods illustrates WSMM's context. Two limitations of WSMM imply the need for a toolkit that overcomes those limitations. An auto rental example is used to illustrate a series of modeling, analysis, and design modules related to different stakeholder purposes. Most of the modules reflect components of Word documents used as outlines to produce over 700 management briefings, mostly by MBA and Executive MBA students, between 2003 and 2017. A concluding section summarizes the main ideas, explains how the use of different modules based on the work system metaphor flexibly supports systems analysis and design, and identifies challenges for future research.
\end{abstract}

\section{KEYWORD}

Enterprise Modeling, Modeling Methods, Systems Analysis and Design, Systems Analysis Toolkit, Work System, Work System Method, Work System Theory

\section{INTRODUCTION}

This research note builds on Alter and Bork (2019), which proposed a work system modeling method (WSMM) that advances a long-term stream of research (e.g., Alter 1995, 2006, 2013) that developed work system theory (WST), the work system method (WSM), and various extensions of WST. Most of that research occurred in the general spirit of design science research (e.g., Rossi et al., 2013) and involved the design and testing of artifacts to be used in analyzing and designing systems. Alter and Bork (2019) was motivated by widely discussed difficulties in the practical application of formal modeling methods that stem from a mismatch between the details and rigor of formal software development techniques and the knowledge, interests, and inclinations of most business professionals. Experienced modeling experts sometimes overcome those difficulties, but even they often find it difficult to elicit full collaboration from business colleagues who lack sophisticated appreciation of modeling.

WSMM attempts to bridge methods designed for different purposes pursued by different stakeholders when moving from rough requirements to programming details. The evolution of WSM

DOI: 10.4018/JDM.2020070101

Copyright $\odot$ 2020, IGI Global. Copying or distributing in print or electronic forms without written permission of IGI Global is prohibited. 
over two decades tried to help business professionals think about systems in terms of business interests and concerns, which does not require high specificity related to technical details. WSMM's attempt to bridge user and developer interests is based on relaxing assumptions about modeling and modeling methods, thereby permitting use of different metamodels to address different modeling purposes with different degrees of formality and specificity. Consistent use of a work system perspective as an invariant modeling metaphor (Ferstl and Sinz, 2013) supports coherence across different purposes.

This research note extends the initial conceptualization of WSMM by proposing an SA\&D toolkit that treats WSMM as part of a broader SA\&D process that includes much more than modeling. The toolkit builds on design artifacts used in over 700 management briefings related to problematic ITreliant work systems produced mostly by MBA and Executive MBA students working individually or in teams between 2003 and 2017.

The next section provides more background by positioning WSMM's central work system metaphor in the context of conceptual modeling, SA\&D, WST, and WSM. A two-dimensional design space for modeling methods illustrates the main idea of WSMM. An SA\&D toolkit proposed here overcomes two important limitations of WSMM. An auto rental example illustrates that toolkit, which includes a series of modeling, analysis, and design modules related to different stakeholder purposes. A concluding section summarizes the main ideas, explains the main implications, notes a practical path to implementation, and identifies challenges for future research.

\section{BACKGROUND}

This section starts with issues related to conceptual models, mentions several modeling perspectives, and then summarizes the work system perspective that is the basis of WSMM.

\section{Conceptual Modeling Issues}

A special issue of the Journal of Database Management on ontological analysis in conceptual modeling (Burton-Jones et al., 2017b) starts with a research commentary in which Wand and Weber $(2017$, p. 2) expresses disappointment that "representation theory (RT), conceptual modeling, and ontology have attracted little attention" from IS researchers despite a belief (shared by the current authors) that conceptual modeling is at the core of the IS discipline. Burton-Jones et al. (2017a) summarizes the main ideas of RT and provides an extensive literature review covering articles that build on RT. Their critique notes, "a tacit assumption that appears to underlie RT is that the usefulness of information systems increases monotonically with increases in their representational faithfulness" (p. 1323).

Past research leads to questioning similar assumptions in the general realm of conceptual modeling, especially due to mismatches between user capabilities and the nature of many modeling tools. Domain experts often perceive business situations in imprecise ways and may or may not be able to capture their knowledge in conceptual models (cf. Bjeković et al., 2014; Figl, 2017; Zur Muehlen and Recker, 2013). Research on modeling method usage (e.g., Fettke, 2009; Mendling et al., 2010) and model comprehension (e.g., Haisjackl et al., 2018; Johannsen et al., 2014; Mendling et al., 2018) illuminates factors that exacerbate those issues. Modeling tools sometimes force users to express themselves using formalisms that are unfamiliar or difficult to use (Wüest et al., 2017)). Modeling methods often do not fit modelers' aptitudes, knowledge, and purposes (Hinkel et al., 2016; Zur Muehlen \& Recker, 2013). Simões et al. (2018) notes that the "lack of intuitiveness of diagrammatic representations and the complementary role of text-based representations has been underlined in recent research." Cognitive load (Sweller, 1994) for stakeholders becomes increasingly important as unfamiliar symbols and icons proliferate. In combination, those issues support the desirability of recognizing that different purposes of different stakeholders imply different needs for specificity and formality - a reality that is reflected in WSMM. 
11 more pages are available in the full version of this document, which may be purchased using the "Add to Cart" button on the product's webpage:

www.igi-global.com/article/systems-analysis-and-designtoolkit-based-on-work-system-theory-and-itsextensions/256845?camid $=4 \mathrm{v} 1$

This title is available in InfoSci-Journals, InfoSci-Journal

Disciplines Business, Administration, and Management, InfoSci-Journal Disciplines Computer Science, Security, and Information Technology, InfoSci-Journal Disciplines Library Science, Information Studies, and Education, InfoSciEducation Knowledge Solutions - Journals, InfoSciComputer Science and IT Knowledge Solutions - Journals, InfoSci-Business Knowledge Solutions - Journals.

Recommend this product to your librarian: www.igi-global.com/e-resources/libraryrecommendation/?id=2

\section{Related Content}

Programming and Pre-Processing Systems for Big Data Storage and Visualization

Hidayat Ur Rahman, Rehan Ullah Khan and Amjad Ali (2018). Handbook of Research on Big Data Storage and Visualization Techniques (pp. 228-253). www.igi-global.com/chapter/programming-and-pre-processing-systems-forbig-data-storage-and-visualization/198765?camid=4v1a 
Transforming Activity-Centric Business Process Models into InformationCentric Models for SOA Solutions

Rong Liu, Frederick Y. Wu and Santhosh Kumaran (2012). Cross-Disciplinary Models and Applications of Database Management: Advancing Approaches (pp. 336-358).

www.igi-global.com/chapter/transforming-activity-centric-businessprocess $/ 63673$ ?camid $=4 \mathrm{v} 1 \mathrm{a}$

Technology-Mediated Synchronous Virtual Education: An Empirical Study Xiaofeng Chen and Keng Siau (2016). Journal of Database Management (pp. 39-63). www.igi-global.com/article/technology-mediated-synchronous-virtualeducation $/ 178635$ ? camid $=4 \mathrm{v} 1 \mathrm{a}$

Matching Attributes across Overlapping Heterogeneous Data Sources Using Mutual Information

Huimin Zhao (2012). Cross-Disciplinary Models and Applications of Database Management: Advancing Approaches (pp. 417-437).

www.igi-global.com/chapter/matching-attributes-across-overlappingheterogeneous/63676?camid=4v1a 